\title{
Kan Replacement of Simplicial Manifolds
}

\author{
CHENCHANG ZHU \\ Mathematisches Institut, Georg-August-Universität Göttingen, Bunsenstraße 3-5, \\ 37073 Göttingen, Germany. e-mail: zhu@uni-math.gwdg.de
}

Received: 22 December 2008 / Revised: 29 June 2009 / Accepted: 30 June 2009

Published online: 26 September 2009 - (C) Springer 2009

\begin{abstract}
We establish a functor Kan from local Kan simplicial manifolds to weak Kan simplicial manifolds. It gives a solution to the problem of extending local Lie groupoids to Lie 2-groupoids.
\end{abstract}

Mathematics Subject Classification (2000). 51H25.

Keywords. fibrant replacement, simplicial manifold, Quillen's small object argument, Kan fibration, local Lie groupoid, extension.

\section{Introduction}

Studying the correspondence between global and infinitesimal symmetries is a classical topic. We call the passage from global symmetries to infinitesimal ones differentiation, and the inverse passage integration. A classical example is the case of Lie groups and Lie algebras,

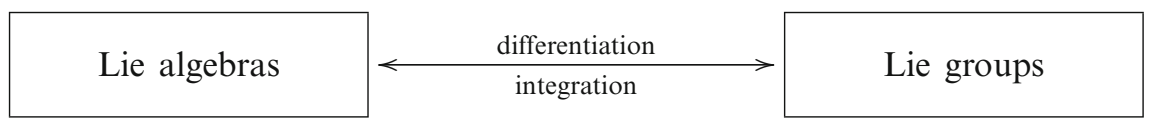

However, when the symmetries become more complicated, such as those of $L_{\infty}$ algebras, or $L_{\infty}$-algebroids, the integration and differentiation both become harder. The following problems have been solved for these higher symmetries: integration of nilpotent $L_{\infty}$-algebras by Getzler [5], integration of general $L_{\infty}$-algebras by Henriques [6], differentiation of $L_{\infty}$-groupoids by Ševera [13], both directions for Lie 1-algebroids by Cattaneo and Felder [2], Crainic and Fernandes [3], and from a higher viewpoint by Tseng and Zhu [11]. Here, the author wants to emphasize a middle step of local symmetries missing in the above correspondence,

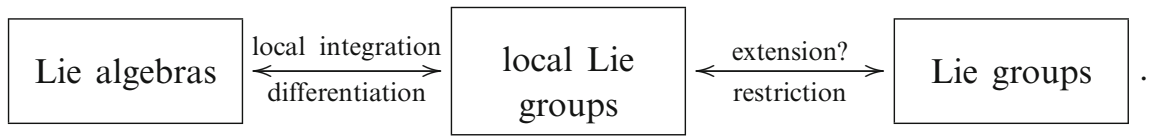

Supported by the German Research Foundation [Deutsche Forschungsgemeinschaft (DFG)] through the Institutional Strategy of the University of Göttingen. 
Indeed, to obtain infinitesimal symmetries by differentiation, we only need local symmetries. Conversely, sometimes it is easier to obtain a local integration, avoiding some analytic issues (for example, in Getzler [5] for $L_{\infty}$-algebras). In this paper, we make our first attempts towards the extension problem from local symmetries to global ones: we construct an extension from local Kan simplicial manifolds to weak Kan ones. The classical extension of a local Lie group to a topological group discussed by van Est [12] can be viewed as the 1-truncation of our result. Its 2-truncation applied to local Lie groupoids provides a solution to the integration problem of Lie algebroids into Lie 2-groupoids [15]. Observe that unlike Lie algebras which are in one-to-one correspondence with simply connected Lie groups, Lie algebroids (integrable or not) are in one-to-one correspondence with Lie 2-groupoids with some étale property.

We use the viewpoint of Kan simplicial manifolds to describe arbitrary Lie $n$-groupoids.

Recall that a simplicial manifold $X$ consists of manifolds $X_{n}$ and structure maps

$$
\begin{aligned}
& d_{i}^{n}: X_{n} \rightarrow X_{n-1} \text { (face maps) } s_{i}^{n}: X_{n} \rightarrow X_{n+1} \text { (degeneracy maps), } \\
& \quad \text { for } i \in\{0,1,2, \ldots, n\}
\end{aligned}
$$

that satisfy suitable coherence conditions (see,e.g., [4]). The first two examples of simplicial manifolds (actually, they are simplicial sets with discrete topology) are the simplicial $m$-simplex $\Delta[m]$ and the horn $\Lambda[m, j]$ with

$$
\begin{aligned}
(\Delta[m])_{n} & =\{f:(0,1, \ldots, n) \rightarrow(0,1, \ldots, m) \mid f(i) \leq f(j), \forall i \leq j\}, \\
(\Lambda[m, j])_{n} & =\left\{f \in(\Delta[m])_{n} \mid\{0, \ldots, j-1, j+1, \ldots, m\} \nsubseteq\{f(0), \ldots, f(n)\}\right\} .
\end{aligned}
$$

The horn $\Lambda[m, j]$ should be thought as a simplicial set obtained from $\Delta[m]$ by taking away its unique non-degenerate $m$-simplex as well as the $j$ th of its $m+1$ non-degenerate $(m-1)$-simplices.

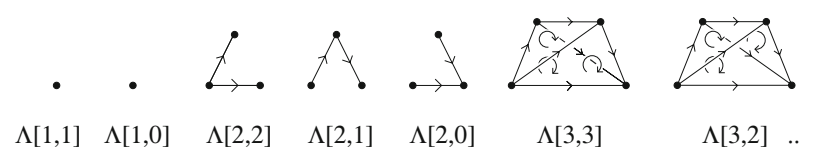

Our convention for arrows is that they are oriented from larger numbers to smaller numbers.

In homotopy theory, the Kan conditions require the natural restriction maps

$$
X_{m}=\operatorname{hom}(\Delta[m], X) \rightarrow \operatorname{hom}(\Lambda[m, j], X),
$$

to be surjective. Equivalently, every horn can be filled up by a simplex. They correspond to the possibility of composing and inverting various morphisms, in the language of groupoids. 
With enrichment in differential geometry, the Kan conditions are

$\operatorname{Kan}(m, j)$ : Equation (3) is a surjective submersion,

$K a n !(m, j)$ : Equation (3) is a diffeomorphism.

But since $\operatorname{hom}(\Lambda[m, j], X)$ is formed by taking the fibre product of the $X_{i}$ 's, it may not be a manifold. However, if Equation (3) is a submersion for all $0 \leq j \leq$ $m \leq m_{0}$, as shown in [6, Lemma 2.4], hom $\left(\Lambda\left[m_{0}, j\right], X\right)$ is a manifold for all $0 \leq$ $j \leq m_{0}$. Hence, we are allowed to define,

DEFINITION 1.1. A Lie n-groupoid $X(n \in \mathbb{N} \cup \infty)$ is a simplicial manifold that satisfies $\operatorname{Kan}(m, j), \forall m \geq 1,0 \leq j \leq m$, and $K a n !(m, j), \forall m>n, 0 \leq j \leq m$. When $n=\infty$, a Lie $\infty$-groupoid is also called a Kan simplicial manifold.

Then a Lie 1-groupoid is simply the nerve of a Lie groupoid.

To describe local Lie groupoids, we need local Kan conditions:

$\operatorname{Kan}^{l}(m, j): \quad$ Equation (3) is a submersion,

$\mathrm{Kan}^{l} !(m, j)$ : Equation (3) is injective étale.

DEFINITION 1.2. A local Lie n-groupoid $X(n \in \mathbb{N} \cup \infty)$ is a simplicial manifold that satisfies $\operatorname{Kan}^{l}(m, j), \forall m \geq 1,0 \leq j \leq m$, and $K_{a n}^{l} !(m, j), \forall m>n, 0 \leq j \leq m$. When $n=\infty$, a local Lie $\infty$-groupoid is also called a local Kan simplicial manifold.

Then, the nerve of a local Lie groupoid is a local Lie 1-groupoid $X$.

As soon as we have done this, it becomes clear that to associate a Kan object $K a n(X)$ to a local Kan simplicial manifold $X$, we need to do some sort of fibrant replacement in the category of simplicial manifolds. However, simplicial manifolds do not form a model category and we need to do it by hand. In fact, the differential category is rather special, even the construction for simplicial presheaves cannot be used directly here. It turns out that the object $K a n(X)$ constructed directly by Quillen's small object argument is not a Kan simplicial manifold, however, it is a simplicial manifold and is Kan as a simplicial set. We also prove certain representability conditions for $\operatorname{Kan}(X)$ that make it into a weak Kan simplicial manifold (see Sect. 2), a property slightly weaker than that of a Kan simplicial manifold. On the other hand, the defects of $K a n(X)$ lie only on high levels, that is, if we perform a 2-truncation $\tau_{2}(\operatorname{Kan}(X))$, and the 2-truncation is still representable, then $\tau_{2}(\operatorname{Kan}(X))$ is indeed a Lie 2-groupoid.

\section{Definition}

To simplify the calculation that we shall encounter later, we introduce a new concept: a simplicial manifold is invertible, if there are isomorphisms

$$
\operatorname{hom}(\Lambda[2,0], X) \cong \operatorname{hom}(\Lambda[2,1], X) \cong \operatorname{hom}(\Lambda[2,2], X),
$$

which are compatible with the face maps $\operatorname{hom}(\Delta[2], X) \rightarrow \operatorname{hom}(\Lambda[2, j], X)$. 
A local Lie groupoid $G_{1}^{\text {loc }} \Rightarrow G_{0}^{\text {loc }}$ has locally defined multiplication $m: V \times{ }_{G_{0}^{\text {loc }}} V$ $\Rightarrow G_{1}^{\text {loc }}$, where $V \subset G_{1}^{\text {loc }}$ is an open neighborhood of $G_{0}^{\text {loc }}$. We can refine $G_{1}^{\text {loc }}$ such that the inverse $i$ is defined from $G_{1}^{\text {loc }}$ to $G_{1}^{\text {loc }}$. Hence, the nerve of a local Lie groupoid is an invertible local Kan simplicial manifold. Since for higher groupoids, we do not have explicit inverses, we drop higher invertibility condition for local Lie $n$-groupoids. In all cases, invertibility is only to simplify the calculation, and our motivation is to deal with local Lie 1-groupoids.

Now, we try to define a functor Kan sending invertible local Kan manifolds to Kan simplicial manifolds by modifying directly Quillen's small object argument. We will see that this construction is not successful, however, we arrive at a simplicial manifold satisfying conditions slightly weaker than the Kan conditions. Let

$$
J:=\{\Lambda[k, j] \rightarrow \Delta[k]: 0 \leq j \leq k \geq 3,\} \cup\{\Lambda[2,1] \rightarrow \Delta[2]\},
$$

be a subset of inclusions with respect to which the Kan conditions have the right lifting property. Given a local Kan manifold $X$, we then construct a series of simplicial manifolds

$$
X=X^{0} \rightarrow X^{1} \rightarrow X^{2} \rightarrow \cdots \rightarrow X^{\beta} \rightarrow \cdots
$$

by an inductive push-out:

$$
\coprod_{(\Lambda[k, j] \rightarrow \Delta[k]) \in J} \Lambda[k, j] \times \operatorname{hom}\left(\Lambda[k, j], X^{\beta}\right) \longrightarrow X_{(\Lambda[k, j] \rightarrow \Delta[k]) \in J} \Delta[k] \times \operatorname{hom}\left(\Lambda[k, j], X^{\beta}\right) \longrightarrow X^{\beta+1}
$$

Then, we let $\operatorname{Kan}(X)=\operatorname{colim}_{\beta \in \mathbb{N}} X^{\beta}$.

Now, we make some calculations for the first steps of this Kan replacement. First of all, $X_{0}=X_{0}^{1}=X_{0}^{2}=\cdots=\operatorname{Kan}(X)_{0}$, and

$$
\begin{aligned}
X_{1}^{1}= & X_{1} \sqcup\left(X_{1} \times_{X_{0}} X_{1}\right) \\
X_{1}^{2}= & X_{1}^{1} \sqcup X_{1}^{1} \times_{X_{0}} X_{1}^{1}= \\
= & X_{1}^{1} \sqcup\left(X_{1} \times_{X_{0}} X_{1} \sqcup X_{1} \times_{X_{0}}\left(X_{1} \times_{X_{0}} X_{1}\right)\right. \\
& \left.\sqcup\left(X_{1} \times_{X_{0}} X_{1}\right) \times_{X_{0}} X_{1} \sqcup\left(X_{1} \times_{X_{0}} X_{1}\right) \times_{X_{0}}\left(X_{1} \times_{X_{0}} X_{1}\right)\right)
\end{aligned}
$$

$$
\operatorname{Kan}(X)_{1}=X_{1} \sqcup\left(X_{1} \times_{X_{0}} X_{1}\right) \sqcup\left(X_{1}^{1} \times_{X_{0}} X_{1}^{1}\right) \sqcup\left(X_{1}^{2} \times_{X_{0}} X_{1}^{2}\right) \ldots,
$$

which we can represent by the following picture:

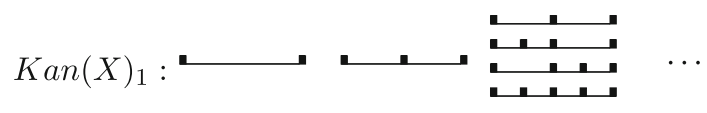


A calculation shows that

$$
\begin{aligned}
X_{2}^{1}= & X_{2} \sqcup X_{1} \times_{X_{0}} X_{1} \sqcup X_{1} \times_{X_{0}} X_{1} \sqcup X_{1} \times_{X_{0}} X_{1} \\
& \sqcup\left(\sqcup_{j=0}^{3} \operatorname{hom}(\Lambda[3, j], X)\right. \\
X_{2}^{2}= & X_{2}^{1} \sqcup X_{1}^{1} \times_{X_{0}} X_{1}^{1} \sqcup X_{1}^{1} \times_{X_{0}} X_{1}^{1} \sqcup X_{1}^{1} \times_{X_{0}} X_{1}^{1} \\
& \sqcup\left(\sqcup_{j=0}^{3} \operatorname{hom}\left(\Lambda[3, j], X^{1}\right)\right.
\end{aligned}
$$

Inside $X_{2}^{1}$, there are three copies of $X_{1} \times_{X_{0}} X_{1}$. The first is an artificial filling of the horn $X_{1} \times X_{0} X_{1}$, and the second two are images of degeneracies of $X_{1} \times X_{0} X_{1}$ in $X_{1}^{1}$. Similarly, for $X_{2}^{2}$, etc., we represent an element in $X_{2}^{1}$ as

$$
X_{2}: \triangle, \quad X_{1} \times_{X_{0}} X_{1}: \cdots, \quad \operatorname{hom}(\Lambda(3, j), X): \quad \Delta \ldots 4 \text { such }
$$

plus the degenerate ones in the other two copies of $X_{1} \times_{X_{0}} X_{1}$. Furthermore, we represent an element in $X_{2}^{2}$ by

$$
\begin{gathered}
X_{2}^{1}: \text { described as above } \\
X_{1}^{1} \times_{X_{0}} X_{1}^{1}: \vdots \vdots \vdots
\end{gathered}
$$

$$
\operatorname{hom}\left(\Lambda[3, j], X^{1}\right) \text { : }
$$
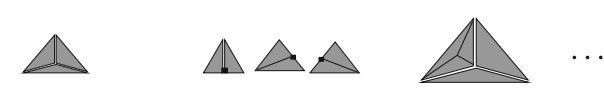

We now show that

$$
X_{2}^{1} \rightarrow \operatorname{hom}\left(\Lambda[2,0], X^{1}\right)=X_{1}^{1} \times_{d_{2}, X_{0}, d_{1}} X_{1}^{1}
$$

is not a submersion.

We first need some technical preparation. A simplicial set $S$ is called collapsible if it admits a filtration

$$
p t=S_{0} \subset S_{1} \subset \cdots \subset S_{k}=S
$$

such that each $S_{i}$ is obtained from the previous one by filling a horn, namely such that $S_{i}$ can be written as $S_{i}=S_{i-1} \sqcup_{\Lambda}\left[n_{i}, l_{i}\right] \Delta\left[n_{i}\right]$ for some injective map $\Lambda\left[n_{i}, l_{i}\right] \hookrightarrow$ $S_{i-1}$. Thus, we can define an order relation on the collapsible simplicial sets: we say that $S$ is not greater than $T$, and we write $S \prec T$, if $T=S_{t}$ and $S=S_{S}$ with $s \leq t$ in Equation (11). By convention, the notation $S \prec T$ also indicates the inclusion map $S \rightarrow T$. We also define the dimension of a collapsible simplicial set $S$ by

$$
\operatorname{dim} S=\max _{k}\{\Delta[k] \prec S\} .
$$

LEMMA 2.1. Given $S \prec T$ and a local Kan simplicial manifold $X$, $\operatorname{hom}(T, X)$ and $\operatorname{hom}(S, X)$ are both manifolds, and the natural map

$$
\operatorname{hom}(T, X) \rightarrow \operatorname{hom}(S, X),
$$

is a submersion. 
This is proven in [6, Lemma 2.4] for Kan simplicial manifolds, but it is easy to see that it works also for local Kan ones since only the submersion condition is used.

We return to the study of the map Equation (10). $X_{2}^{1}$ has several components, and the horn projection map Equation (10) induces on each component the following maps:

$$
\begin{aligned}
X_{2} & \rightarrow \operatorname{hom}(\Lambda[2,0], X), \quad \operatorname{hom}(\Lambda[3, j], X) \rightarrow \operatorname{hom}(\Lambda[2,0], X) \\
X_{1} \times X_{0} X_{1} & \rightarrow X_{1} \times X_{0}\left(X_{1} \times X_{0} X_{1}\right), \quad X_{1} \times_{X_{0}} X_{1} \rightarrow\left(X_{1} \times_{X_{0}} X_{1}\right) \times_{X_{0}}\left(X_{1} \times_{X_{0}} X_{1}\right), \\
X_{1} & \times_{X_{0}} X_{1} \rightarrow X_{1} \times_{X_{0}} X_{1} .
\end{aligned}
$$

The morphisms in Equation (12) are always submersions by Lemma 2.1. However, the morphisms in Equation (13) are not submersions. This implies that $\operatorname{hom}(\Delta[2], \operatorname{Kan}(X)) \rightarrow \operatorname{hom}(\Lambda[2,0], \operatorname{Kan}(X))$ will not be a submersion. Hence, $K a n(X)$ will not be a Kan simplicial manifold, but we will prove that it satisfies

(A) $\operatorname{Kan}(X)$ is a simplicial manifold;

(B) $\operatorname{hom}(S, \operatorname{Kan}(X))$ is a manifold for any collapsible $S$;

(C) the natural map $\operatorname{Kan}(X)_{n} \rightarrow \operatorname{hom}(\Lambda[n, l], \operatorname{Kan}(X))$ is surjective for all $n$ and $l$ with $0 \leq l \leq n$.

Simplicial manifolds satisfying such conditions are called weak Kan simplicial manifolds. These weak Kan simplicial manifolds are indeed Kan as simplicial sets. The submersion condition in the Kan conditions is replaced by condition (B). By Lemma 2.1, we can see that the submersion condition implies (B), hence weak Kan is indeed weaker than Kan. However, a submersion condition is usually applied to guarantee some representability, for example, the one in condition (B). Hence, we see that in many cases this condition will be sufficient to conclude. For example, we can still talk about hypercovers of weak Kan simplicial manifolds (but they will not be used in this paper).

Before attacking the problem, we prove a technical lemma:

LEMMA 2.2. Suppose that the $X^{\beta}$ 's are a sequence of simplicial sets constructed by Equation (6).

1. If $S$ is a collapsible simplicial set, then we can decompose

$$
\operatorname{hom}\left(S, X^{\beta+1}\right)=\sqcup_{a \in A} \operatorname{hom}\left(S_{a}, X^{\beta}\right),
$$

with a finite set of collapsible simplicial sets $\left\{S_{a}: a \in A\right\} \ni S$ satisfying $\operatorname{dim} S_{a} \leq$ $\operatorname{dim} S$.

2. This decomposition respects morphisms, that is if $S \prec T$, and both of them have a decomposition,

$$
\operatorname{hom}\left(S, X^{\beta+1}\right)=\sqcup_{a \in A} \operatorname{hom}\left(S_{a}, X^{\beta}\right), \quad \operatorname{hom}\left(T, X^{\beta+1}\right)=\sqcup_{a^{\prime} \in A^{\prime}} \operatorname{hom}\left(T_{a^{\prime}}, X^{\beta}\right),
$$


then there is a map $\alpha: A^{\prime} \rightarrow A$, and morphisms of simplicial sets $S_{\alpha\left(a^{\prime}\right)} \rightarrow T_{a^{\prime}}$, such that the natural morphism $\operatorname{hom}\left(T, X^{\beta+1}\right) \rightarrow \operatorname{hom}\left(S, X^{\beta+1}\right)$ is induced from $\operatorname{hom}\left(T_{a^{\prime}}, X^{\beta}\right) \rightarrow \operatorname{hom}\left(S_{\alpha\left(a^{\prime}\right)}, X^{\beta}\right)$ on the level of their decompositions.

Proof. Since the procedure to form $X^{\beta+1}$ from $X^{\beta}$ is the same as the one to form $X^{1}$ from $X$, we only have to prove the two statements for $\beta=0$. Since $X$ is arbitrary, the decomposition in Equation (1) is clearly unique. We use an induction on the size of $S$ and $T$. The initial assumption is verified in the calculation we did earlier in this section. It is clear that Equation (1) holds for $\Delta[m]$ for $m \in \mathbb{N}$. Now with a fixed $n$, we consider a horn filling diagram as we mentioned in the procedure of Equation (11)

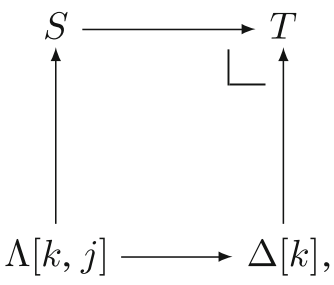

with $\operatorname{dim} S \leq \operatorname{dim} T \leq n-1$, and $k \leq n-1$.

We suppose that

(i) statement (1) is true for all $S^{\prime}$ with $S^{\prime} \prec S$;

(ii) statement (2) is true for $S^{\prime} \prec T^{\prime}$ and $S^{\prime} \prec \Delta[n]$ when $\operatorname{dim} S^{\prime} \leq \operatorname{dim} T^{\prime} \leq n-1$ and when (1) is true for $S^{\prime}$ and $T^{\prime}$.

To complete the induction, we will prove that

- statement (1) holds for $T$;

- statement (2) holds for $S \prec T$, and holds for $T \prec \Delta[n]$ if such a map $T \rightarrow \Delta[n]$ exists.

First, we apply hom $\left(-, X^{1}\right)$ to Equation (15) and apply the induction hypothesis to $\operatorname{hom}\left(S, X^{1}\right), \operatorname{hom}\left(\Lambda[k, j], X^{1}\right)$ and $\operatorname{hom}\left(\Delta[k], X^{1}\right)$. Then we have

$$
\begin{aligned}
\operatorname{hom}\left(T, X^{1}\right) & =\operatorname{hom}\left(S, X^{1}\right) \times_{\operatorname{hom}\left(\Lambda[k, j], X^{1}\right)} \operatorname{hom}\left(\Delta[k], X^{1}\right)= \\
& =\sqcup_{a^{\prime} \in A^{\prime}} \operatorname{hom}\left(S_{a^{\prime}}^{\prime}, X\right) \times_{\sqcup_{a \in A}} \operatorname{hom}\left(S_{a}, X\right) \sqcup_{a^{\prime \prime} \in A^{\prime \prime}} \operatorname{hom}\left(S_{a^{\prime \prime}}^{\prime \prime}, X\right)= \\
& =\sqcup_{b \in B} \operatorname{hom}\left(T_{b}, X\right) .
\end{aligned}
$$

Here, $T_{b}$ is formed when $\alpha\left(a^{\prime}\right)=\alpha\left(a^{\prime \prime}\right)$ by

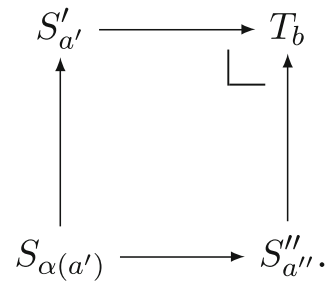


We obtain a map $B \rightarrow A^{\prime}$ defined by $b \mapsto a^{\prime}$ and morphisms $S_{a^{\prime}}^{\prime} \rightarrow T_{b}$. They induce the morphisms $\operatorname{hom}\left(T_{b}, X\right) \rightarrow \operatorname{hom}\left(S_{a^{\prime}}^{\prime}, X\right)$, hence the morphism $\operatorname{hom}\left(T, X^{1}\right) \rightarrow$ $\operatorname{hom}\left(S, X^{1}\right)$. It's not hard to see that $T \in\left\{T_{b}\right\}$ by the induction hypothesis and Equation (15).

Suppose hom $\left(\Delta[n], X^{1}\right)=\sqcup_{c \in C}\left(D_{c}, X\right)$. If there is a map $T \prec \Delta[n]$, by restriction, we obtain maps $S \prec \Delta[n], \Lambda[k, j] \prec \Delta[n]$, and $\Delta[k] \prec \Delta[n]$ which fit in the following commutative diagram:

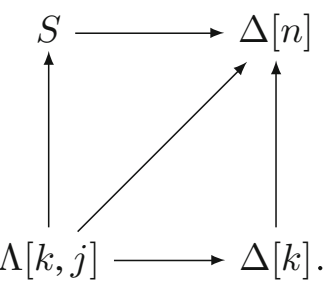

By the induction hypothesis, we have

- the morphism $\operatorname{hom}\left(\Delta[n], X^{1}\right) \rightarrow \operatorname{hom}\left(S, X^{1}\right)$ is induced by a map $\alpha^{\prime}: C \rightarrow A^{\prime}$ and morphisms $S_{\alpha^{\prime}(c)}^{\prime} \rightarrow D_{c}$;

- the morphism $\operatorname{hom}\left(\Delta[n], X^{1}\right) \rightarrow \operatorname{hom}\left(\Lambda[k, j], X^{1}\right)$ is induced by a map $\alpha: C \rightarrow$ $A$ and morphisms $S_{\alpha(c)} \rightarrow D_{c}$;

- the morphism $\operatorname{hom}\left(\Delta[n], X^{1}\right) \rightarrow \operatorname{hom}\left(\Delta[k], X^{1}\right)$ is induced by a map $\alpha^{\prime \prime}: C \rightarrow$ $A^{\prime \prime}$ and morphisms $S_{\alpha^{\prime \prime}(c)}^{\prime \prime} \rightarrow D_{c}$.

We see that $\operatorname{hom}\left(D_{c}, X\right) \rightarrow \operatorname{hom}\left(S_{\alpha(c)}, X\right)$ induces $\operatorname{hom}\left(\Delta[n], X^{1}\right) \rightarrow \operatorname{hom}(\Lambda[k, j]$, $\left.X^{1}\right)$, and the composed morphism $\operatorname{hom}\left(D_{c}, X\right) \rightarrow \operatorname{hom}\left(S_{\alpha^{\prime}(c)}^{\prime}, X\right) \rightarrow \operatorname{hom}\left(S_{\alpha\left(\alpha^{\prime}(c)\right)}, X\right)$ induces $\operatorname{hom}\left(\Delta[n], X^{1}\right) \rightarrow \operatorname{hom}\left(S, X^{1}\right) \rightarrow \operatorname{hom}\left(\Lambda[k, j], X^{1}\right)$, which is the same morphism as $\operatorname{hom}\left(\Delta[n], X^{1}\right) \rightarrow \operatorname{hom}\left(\Lambda[k, j], X^{1}\right)$. Hence by the uniqueness of the decomposition, we have $\alpha\left(\alpha^{\prime}(c)\right)=\alpha(c)$ and similarly $\alpha(c)=\alpha\left(\alpha^{\prime \prime}(c)\right)$, and a commutative diagram,

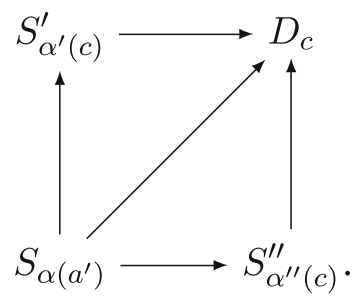

Then $T_{b(c)}$ defined by the push-out diagram,

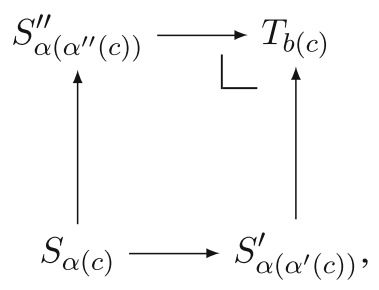


has a canonical map $T_{b(c)} \rightarrow D_{c}$, where $b(c)$ is an index in $B$ and depends only on $c$. Since $\operatorname{hom}\left(T, X^{1}\right)$ is a fibre product, these canonical maps induce the map $\operatorname{hom}\left(\Delta[n], X^{1}\right) \rightarrow \operatorname{hom}\left(T, X^{1}\right)$ via the maps $\operatorname{hom}\left(D_{c}, X\right) \rightarrow \operatorname{hom}\left(T_{b(c)}, X\right)$.

PROPOSITION-DEFINITION 2.3. The operation Kan constructed in Equation (6) is a functor from the category of local Kan manifolds $X$ to the category of weak Kan simplicial manifolds.

Proof. The construction of Kan makes it clear that it is functorial. Since $\operatorname{Kan}(X)=\operatorname{colim}_{\beta} X^{\beta}$, given any finite simplicial set $A$ (a collapsible simplicial set $S$ is such), the natural map of sets is an isomorphism,

$$
\operatorname{colim}_{\beta} \operatorname{hom}\left(A, X^{\beta}\right) \stackrel{\simeq}{\rightarrow} \operatorname{hom}(A, \operatorname{Kan}(X)) .
$$

Moreover, by Lemma 2.2,

$$
\operatorname{hom}\left(S, X^{\beta+1}\right)=\operatorname{hom}\left(S, X^{\beta}\right) \bigsqcup\left(\sqcup_{a} \operatorname{hom}\left(S_{a}, X^{\beta}\right)\right) .
$$

We then use Lemma 2.2 recursively, and obtain that for any collapsible simplicial set $T$,

$$
\operatorname{hom}\left(T, X^{\beta}\right)=\sqcup \operatorname{hom}\left(T_{p}, X\right),
$$

for a finite set of collapsible simplicial sets $T_{p}$. Hence, $\operatorname{hom}\left(S, X^{\beta}\right)$ and $\operatorname{hom}\left(S_{a}\right.$, $X^{\beta}$ ) are manifolds because $X$ is a local Kan manifold. By Equations (17) and (16), $\operatorname{hom}(S, \operatorname{Kan}(X))$ is a disjoint union of manifolds.

Therefore, it remains to show that $\operatorname{Kan}(X)$ is Kan as a simplicial set. We take an element $A \rightarrow B$ of $J$ and a solid arrow diagram,

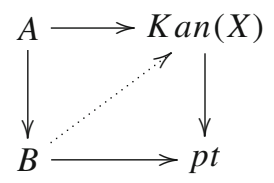

then we must show that the dotted arrow exists. By the isomorphism Equation (16), the map $A \rightarrow \operatorname{Kan}(X)$ factors through $X^{\beta} \rightarrow \operatorname{Kan}(X)$ for some $\beta$ and we have the solid arrow diagram

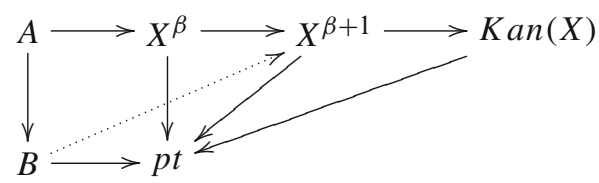

Since $X^{\beta+1}$ is constructed as the push-out in Equation (6), the dotted arrow naturally exists, and this dotted arrow defines the one in Equation (18). 
Now, we only have to verify that the dotted arrow in Equation (18) exists for $\Lambda[1, j] \rightarrow \Delta[1]$ for $j=0,1$ and $\Lambda[2, j] \rightarrow \Delta[2]$ for $j=0,2$. We have $X_{0}^{\beta}=X_{0}$, and

$\operatorname{hom}\left(\Lambda[1, j], X^{\beta+1}\right)=X_{0}^{\beta+1}=X_{0}, \quad \operatorname{hom}\left(\Delta[1], X^{\beta+1}\right)=X_{1}^{\beta} \times_{d_{0}, X_{0}, d_{1}} X_{1}^{\beta}$,

thus the map $\operatorname{hom}\left(\Delta[1], X^{\beta+1}\right) \rightarrow \operatorname{hom}\left(\Lambda[1, j], X^{\beta+1}\right)$ being the pull-back of $d_{1}$ or $d_{0}$, has to be a surjective submersion. Now, we prove that if $X^{\beta}$ is invertible, then $X^{\beta+1}$ is also invertible. In fact,

$$
\begin{aligned}
\operatorname{hom}\left(\Lambda[2,2], X^{\beta+1}\right)= & X_{1}^{\beta+1} \times_{d_{1}, X_{0}, d_{1}} X_{1}^{\beta+1}= \\
= & \left(X_{1}^{\beta} \sqcup X_{1}^{\beta} \times_{d_{0}, X_{0}, d_{1}} X_{1}^{\beta}\right) \times_{d_{1}, X_{0}, d_{1}}\left(X_{1}^{\beta} \sqcup X_{1}^{\beta} \times{ }_{d_{0}, X_{0}, d_{1}} X_{1}^{\beta}\right)= \\
= & X_{1}^{\beta} \times_{d_{1}, X_{0}, d_{1}} X_{1}^{\beta} \sqcup X_{1}^{\beta} \times_{d_{1}, X_{0}, d_{1}}\left(X_{1}^{\beta} \times{ }_{d_{0}, X_{0}, d_{1}} X_{1}^{\beta}\right) \\
& \sqcup\left(X_{1}^{\beta} \times_{d_{0}, X_{0}, d_{1}} X_{1}^{\beta}\right) \times_{d_{1}, X_{0}, d_{1}} X_{1}^{\beta} \sqcup \ldots
\end{aligned}
$$

Since $X^{\beta}$ is invertible, $X_{1}^{\beta} \times_{d_{1}, X_{0}, d_{1}} X_{1}^{\beta} \cong X_{1}^{\beta} \times_{d_{0}, X_{0}, d_{1}} X_{1}^{\beta}$. Hence

$$
\begin{aligned}
X_{1}^{\beta} \times{ }_{d_{1}, X_{0}, d_{1}}\left(X_{1}^{\beta} \times \times_{d_{0}, X_{0}, d_{1}} X_{1}^{\beta}\right) & \cong X_{1}^{\beta} \times_{d_{0}, X_{0}, d_{1}} X_{1}^{\beta} \times_{d_{0}, X_{0}, d_{1}} X_{1}^{\beta}, \\
\left(X_{1}^{\beta} \times{ }_{d_{0}, X_{0}, d_{1}} X_{1}^{\beta}\right) \times_{d_{1}, X_{0}, d_{1}} X_{1}^{\beta} & \cong X_{1}^{\beta} \times_{d_{1}, X_{0}, d_{1}} X_{1}^{\beta} \times_{d_{0}, X_{0}, d_{1}} X_{1}^{\beta} \cong \\
& \cong\left(X_{1}^{\beta} \times_{d_{0}, X_{0}, d_{1}} X_{1}^{\beta}\right) \times_{d_{0}, X_{0}, d_{1}} X_{1}^{\beta},
\end{aligned}
$$

It is easy to continue to verify that $X^{\beta+1}$ is invertible. Then the final result follows from Equation (16).

Given an invertible local Kan manifold $X$, we call $\operatorname{Kan}(X)$ the Kan replacement of $X$.

Even though $\operatorname{Kan}(X)$ is not Kan, its 2-truncation $\tau_{2}(\operatorname{Kan}(X))$ behaves well. We define the $n$-truncation $\tau_{n}$ (it was denoted by $\tau_{\leq n}$ in [6, Section 3]), of a simplicial manifold $X$ by

$$
\tau_{n}(X)_{k}=X_{k}, \quad \forall k \leq n-1, \quad \tau_{n}(X)_{k}=X_{k} / \sim_{k}, \quad \forall k \geq n,
$$

where two elements $x \sim_{k} y$ in $X_{k}$ if they are homotopic ${ }^{1}$ and have the same $n$-skeleton. Since in the process, taking a quotient is involved, the result $\tau_{n}(X)$ might not be a simplicial manifold anymore. We view it as a simplicial stack. When $X$ is $\operatorname{Kan}, \tau_{n}(X)$ viewed as a simplicial set is always a discrete $n$-groupoid. It is representable, namely it is indeed a simplicial manifold with the quotient topology, if and only if the quotient $X_{n} / \sim_{n}$ is representable because the higher levels are decided by $X_{n} / \sim_{n}$. Even though $\operatorname{Kan}(X)$ is not a Kan manifold, we still have

${ }^{1}$ This means that $d_{i} x=d_{i} y, 0 \leq i \leq k$, and there exists $z \in X_{k+1}$ such that $d_{k}(z)=x, d_{k+1}(z)=y$, and $d_{i} z=s_{k-1} d_{i} x=s_{k-1} d_{i} y, 0 \leq i<k$. 
PROPOSITION 2.4. When $\operatorname{Kan}(X)_{2} / \sim_{2}$ is representable, $\tau_{2}(\operatorname{Kan}(X))$ is a Lie 2-groupoid.

Proof. As a simplicial set, $\operatorname{Kan}(X)$ is Kan. Hence $\operatorname{hom}\left(\Delta[n], \tau_{2}(\operatorname{Kan}(X))\right) \cong$ $\operatorname{hom}\left(\Lambda[n, j], \tau_{2}(\operatorname{Kan}(X))\right)$, for $n \geq 3$. Especially, $\tau_{2}(\operatorname{Kan}(X))_{3} \cong \operatorname{hom}(\Lambda[3,0]$, $\left.\tau_{2}(\operatorname{Kan}(X))\right)$. Since the higher layers are determined by the first four layers,

$$
\tau_{2}(\operatorname{Kan}(X))=\operatorname{Cosk}^{3} \circ \operatorname{Sk}^{3}\left(\tau_{2}(\operatorname{Kan}(X))\right),
$$

by the same argument as in [16, Section 2.3], to show that $\tau_{2}(\operatorname{Kan}(X))$ is a Lie 2-groupoid, we only need to show that $\operatorname{hom}\left(\Lambda[3,0], \tau_{2}(\operatorname{Kan}(X))\right)$ is representable and that $\tau_{2}(\operatorname{Kan}(X))$ satisfies the condition $\operatorname{Kan}(m, j)$ for $m \leq 2$. In fact, the induction argument there already shows that the representability of $\operatorname{hom}\left(\Lambda[3,0], \tau_{2}(\operatorname{Kan}(X))\right)$ is implied by the fact that $\tau_{2}(\operatorname{Kan}(X))$ satisfies $\operatorname{Kan}(m, j)$ for $m \leq 2$ since $\tau_{2}(\operatorname{Kan}(X))_{2}=\operatorname{Kan}(X)_{2} / \sim_{2}$ is representable. Hence, we only need to show $\operatorname{Kan}(m \leq 2, j)$.

As shown in Definition-Proposition 2.3, hom $\left(\Delta[1], X^{\beta+1}\right) \rightarrow \operatorname{hom}\left(\Lambda[1, j], X^{\beta+1}\right)$ being the pull-back of $d_{1}$ or $d_{0}$, is a surjective submersion, hence $\operatorname{hom}(\Delta[1]$, $\operatorname{Kan}(X)) \rightarrow \operatorname{hom}(\Lambda[1, j], \operatorname{Kan}(X))$ is a surjective submersion. This is $\operatorname{Kan}(1, j)$ for $\operatorname{Kan}(X)$, hence for $\tau_{2}(\operatorname{Kan}(X))$.

The surjectivity property in $\operatorname{Kan}(2, j)$ is automatically satisfied. In fact, since $\operatorname{Kan}(X)$ is Kan as a simplicial set, the composed map

$$
\operatorname{Kan}(X)_{2} \rightarrow \tau_{2}(\operatorname{Kan}(X)) \stackrel{p}{\rightarrow} \operatorname{hom}(\Lambda[2, j], \operatorname{Kan}(X))=\operatorname{hom}\left(\Lambda[2, j], \tau_{2}(\operatorname{Kan}(X))\right),
$$

is surjective, hence the desired map $p$ is also surjective. We only need to show the submersion part. Then what happened to the points where the horn projection map of $\operatorname{Kan}(X)_{2}$ is not a submersion? These points necessarily represent degenerate faces. In fact, an element $\eta \in \operatorname{Kan}(X)_{2}$ can be described as a tree as stated in Lemma 3.8. If all the vertices of the tree are triangles in $X_{2}$, then the horn projection map is a submersion for $\operatorname{Kan}(X)_{2}$, hence $p$ is a submersion. The problem happens exactly when the tree contains at least one vertex coming from one of the three copies of $X_{1} \times_{X_{0}} X_{1}$. But these bad pieces as in Equations (13) and (14) are all homotopic via elements in $\operatorname{Kan}(X)_{3}$ to the boundary of good pieces as in Equation (12), where the submersion holds. Hence, the submersion property also is true for the 2-truncation.

\section{Universal Properties}

Given a local Lie 1-groupoid $W$ (or the nerve of a local Lie groupoid), it extends to a Lie 2-groupoid $\tau_{2}(\operatorname{Kan}(W))$. In [15], we verified that $\tau_{2}(\operatorname{Kan}(W))$ is always a Lie 2-groupoid (even though $\tau_{1}(K a n(W))$ might not be Lie) with a universal property. 
For this purpose, we need to show some universal properties of our Kan replacement. It should be stable under Morita equivalence of simplicial manifolds (and this needs to be made precise), and if some simplicial manifold $X$ is Kan, then $\operatorname{Kan}(X)$ should be Morita equivalent to $X$. We begin by introducing concepts related to Morita equivalence.

\subsection{MORITA EQUIVALENCE OF LOCAL KAN MANIFOLDS}

The reader's first guess is probably that a morphism $f: X \rightarrow Y$ of simplicial manifolds ought to be a simplicial smooth map, i.e., a collection of smooth maps $f_{n}$ : $X_{n} \rightarrow Y_{n}$ that commute with faces and degeneracies. We shall call such a morphism a strict map from $X$ to $Y$. Unfortunately, it is known that, already in the case of usual Lie groupoids, such a notion is not adequate. Indeed there are strict maps that are not invertible even though they deserve to be called isomorphisms; here is an example of such a situation. Consider a manifold $M$ with an open cover $\left\{\mathcal{U}_{\alpha}\right\}$. There is a natural map from the simplicial manifold $X$ with $X_{n}=\bigsqcup_{\alpha_{1}, \ldots, \alpha_{n}} \mathcal{U}_{\alpha_{1}} \cap$ $\cdots \cap \mathcal{U}_{\alpha_{n}}$ to the constant simplicial manifold $M$. All the fibers of this map are simplices, in particular, they are contractible simplicial sets. Nevertheless, this map has no inverse. That is why the notion of Hilsum-Skandalis bimodule [9] was introduced.

The second guess is then to define a special class of strict maps which we shall call hypercovers. A map from $X$ to $Y$ would then be a zig-zag of strict maps $X \stackrel{\sim}{\leftarrow}$ $Z \rightarrow Y$, where the map $Z \rightarrow X$ is a hypercover.

Another, equivalent, to define a generalized morphism of simplicial manifolds uses the Cartesian fibrations of [7, Section 2.4]. In this paper, we use the zig-zag method with the notion of hypercover.

Our definition of hypercover is inspired by the notion of hypercover of étale simplicial objects [1,4] and by Quillen's trivial fibrations for simplicial sets [10].

Recall that [8, Section I.3], given a pointed Kan simplicial set $X$, i.e., $X_{0}=p t$, its homotopy groups are given by

$$
\pi_{n}(X):=\left\{x \in X_{n} \mid d_{i}(x)=p t \text { for all } i\right\} / \sim
$$

where $x \sim x^{\prime}$ if there exists an element $y \in X_{n+1}$ such that $d_{0}(y)=x, d_{1}(y)=x^{\prime}$, and $d_{i}(y)=p t$ for all $i>1$. In general, when $X_{0}$ is not necessarily a point, $\pi_{n}$ is a sheaf over $X_{0}$.

LEMMA 3.1. Given a map $S \rightarrow T$ of pointed Kan simplicial sets, if for any $n \geq 0$ and any commutative solid arrow diagram

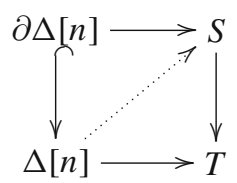


there exists a dotted arrow that makes both triangles commute, then this map is a homotopy equivalence, i.e., $\pi_{n}(S)=\pi_{n}(T)$. Here, $\partial \Delta[n]$ stands for the boundary of the n-simplex.

The proof is standard.

Translating the condition of Lemma 3.1 into a condition on the corresponding hom spaces, we obtain,

DEFINITION 3.2. A strict map $f: Z \rightarrow X$ of local Kan simplicial manifolds is a hypercover if the natural map

$$
Z_{m}=\operatorname{hom}(\Delta[m], Z) \rightarrow \operatorname{hom}(\partial \Delta[m] \rightarrow \Delta[m], Z \rightarrow X)
$$

is a surjectve submersion for all $m \geq 0$.

Here, $\operatorname{hom}(A \rightarrow B, Z \rightarrow X)$ denotes the pull-back spaces of the form $\operatorname{hom}(A, Z) \times \operatorname{hom}(A, X) \operatorname{hom}(B, X)$, where the maps are induced by some fixed maps $A \rightarrow B$ and $Z \rightarrow X$. The notation indicates that this space parameterizes all commuting diagrams of the form<smiles>[Y]1=[Y]C[X]=[X]1</smiles>

where we allow the horizontal arrows to vary but we fix the vertical ones.

Similarly, we can define hypercovers for Lie $n$-groupoids:

DEFINITION 3.3. A strict map $f: Z \rightarrow X$ of Lie n-groupoids is a hypercover if the natural map Equation (20) is a surjective submersion for all $0 \leq m<n$ and is an isomorphism when $m=n$.

Remark 3.4. As proved in [14], if $f: Z \rightarrow X$ is a hypercover of Lie $n$-groupoids, then Equation (20) is automatically an isomorphism for all $m>n$.

As in the case of Definition 1.1, we need to justify that the pull-back $\operatorname{hom}(\partial \Delta[m] \rightarrow \Delta[m], Z \rightarrow X)$ is a manifold. This is rather surprising since the spaces hom $(\partial \Delta[m], Z)$ need not be manifolds (for example take $m=2$ and $Z$ the cross product groupoid associated to the action of $S^{1}$ on $\mathbb{R}^{2}$ by rotation around the origin). We justified this in [14] for Kan simplicial manifolds, but it is clear that only the submersion property is needed, hence the same proof is valid for local Kan manifolds.

DEFINITION 3.5. Two local Kan simplicial manifolds $X$ and $Y$ are Morita equivalent if there exists a local Kan simplicial manifold $Z$ such that both maps $X \stackrel{\sim}{\leftarrow}$ 
$Z \stackrel{\sim}{\rightarrow} Y$ are hypercovers. We call the equivalence relation thus defined [14, Section 2] the Morita equivalence of local Kan simplicial manifolds.

We also define Morita equivalence of Lie $n$-groupoids in the same fashion using hypercovers of Lie $n$-groupoids.

Hypercovers of Lie $n$-groupoids may also be understood as higher analogues of pull-backs of Lie groupoids. Let $X$ be a 2-groupoid and let $Z_{1} \Rightarrow Z_{0}$ be two manifolds with structure maps as in Equation (1) up to the level $n=1$, and let $f_{n}$ : $Z_{n} \rightarrow X_{n}$ be a map preserving the structure maps $d_{k}^{n}$ 's and $s_{k}^{n-1}$ 's for $n \leq 1$. Then, $\operatorname{hom}(\partial \Delta[n], Z)$ is defined for $n \leq 1$. We further suppose that $f_{0}: Z_{0} \rightarrow X_{0}$ is a surjective submersion (hence $Z_{0} \times Z_{0} \times X_{0} \times X_{0} X_{1}$ is a manifold) and $Z_{1} \rightarrow Z_{0} \times Z_{0} \times X_{0} \times X_{0}$ $X_{1}$ also, that is, to say we suppose that the induced maps from $Z_{k}$ to the pullbacks $\operatorname{hom}(\partial \Delta[k], Z) \times_{\operatorname{hom}(\partial \Delta[k], X)} X_{k}$ are surjective submersions for $k=0,1$. Then, we form

$$
Z_{2}=\operatorname{hom}(\partial \Delta[2], Z) \times_{\operatorname{hom}(\partial \Delta[2], X)} X_{2},
$$

which is a manifold (see [14, Lemma 2.4]).

Moreover there are maps $d_{i}^{2}: Z_{2} \rightarrow Z_{1}$ induced by the natural projections $\operatorname{hom}(\partial \Delta[2], Z) \rightarrow Z_{1}$, and maps $s_{i}^{1}: Z_{1} \rightarrow Z_{2}$ defined by

$$
s_{0}^{1}(h)=\left(h, h, s_{0}^{0}\left(d_{0}^{1}(h)\right), s_{0}^{1}\left(f_{1}(h)\right)\right), \quad s_{1}^{1}(h)=\left(s_{0}^{0}\left(d_{1}^{1}(h)\right), h, h, s_{1}^{1}\left(f_{1}(h)\right)\right),
$$

and maps $m_{i}: \operatorname{hom}(\Lambda[3, i], Z) \rightarrow Z_{2}$ with

$$
m_{0}\left(\left(h_{2}, h_{5}, h_{3}, \bar{\eta}_{1}\right),\left(h_{4}, h_{5}, h_{0}, \bar{\eta}_{2}\right),\left(h_{1}, h_{3}, h_{0}, \bar{\eta}_{3}\right)\right)=\left(h_{2}, h_{4}, h_{1}, m_{0}\left(\bar{\eta}_{1}, \bar{\eta}_{2}, \bar{\eta}_{3}\right)\right),
$$

and similarly for the other $m_{i}$ 's.

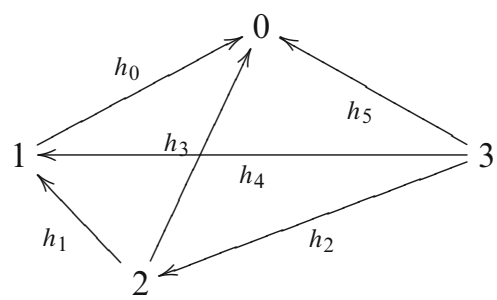

Then $Z_{2} \Rightarrow Z_{1} \Rightarrow Z_{0}$ is a Lie 2-groupoid and we call it the pull-back 2-groupoid of $X$ by $f$. Then $f: Z \rightarrow X$ is a hypercover with $f_{2}$ the natural projection $f_{2}: Z_{2} \rightarrow X_{2}$.

\subsection{THEOREMS AND LEMMAS}

We shall prove that if $X$ is a Kan simplicial manifold, then there is a Morita equivalence $X \stackrel{\sim}{\leftrightarrow} \operatorname{Kan}(X)$. It is very easy to prove this result for simplicial sets. Since the procedure of Kan replacement is basically to fill out horns, the geometric realizations of $\operatorname{Kan}(X)$ and $X$ are homotopic. Since $X$ is Kan, the geometric realizations of $\operatorname{Kan}(X)$ and $X$ being homotopic is equivalent to the fact that the natural embedding map $X \rightarrow \operatorname{Kan}(X)$ satisfies the lifting property described in 
Equation (19). However, the tool of homotopy theory which is available for simplicial sets cannot be used for simplicial manifolds in general. In fact, these do not form a model category, and building a certain machinery as a suitable replacement for model categories should be the correct method of proving the following theorems and lemmas. In the case of simplicial sets, one can easily obtain a morphism $\pi: \operatorname{Kan}(X) \rightarrow X$ such that the composition $X \rightarrow \operatorname{Kan}(X) \rightarrow X$ is the identity. Then, it is straightforward to check that $\operatorname{Kan}(X) \rightarrow X$ has the correct lifting property. However, $\pi$ is not unique (it depends on the choice of fillings in the Kan condition). Hence when generalized to a differential category, $\pi$ is not a continuous morphism in general. This forces us to use another proof. Here, we provide a proof for Lie 2-groupoids $W$.

THEOREM 3.6. If $W$ is a Lie 2-groupoid, then $\tau_{2}(\operatorname{Kan}(W))$ is a Lie 2-groupoid which is Morita equivalent to $W$.

Proof. In general, there is no direct map from $\tau_{2}(\operatorname{Kan}(W))$ to $W$ because there is no (unique) multiplication map $W_{1} \times W_{0} W_{1} \rightarrow W_{1}$. Hence, we must construct a middle step.

A more natural way to describe this construction is to use the corresponding stacky groupoid $\mathcal{G} \Rightarrow W_{0}$, where $\mathcal{G}$ is presented by the Lie groupoid $G_{1} \Rightarrow G_{0}$, with $G_{0}=W_{1}$ and $G_{1}$ the set of bi-gons in $W_{2}$, and the multiplication $\mathcal{G} \times W_{0} \mathcal{G} \rightarrow \mathcal{G}$ is presented by the bimodule $E_{m}=W_{2}$. The bimodules of various compositions of multiplication from various copies of $\mathcal{G}$ to $\mathcal{G}$ are presented by various fibre products of $W_{2}$ 's. For example, the bimodule $W_{2} \times_{d_{1}, W_{1}, d_{2}} W_{2}$, with the moment maps $J_{l}$ to $W_{1} \times W_{0} W_{1} \times W_{0} W_{1}$ and $J_{r}$ to $W_{1}$, presents the multiplication

$$
m \circ(m \times i d):\left(\mathcal{G} \times{ }_{W_{0}} \mathcal{G}\right) \times{ }_{W_{0}} \mathcal{G} \rightarrow \mathcal{G} .
$$

To simplify the notation, we denote a $k$-fold fibre product by $\square^{\times k}$ when no confusion can arise. We let $Z_{0}=W_{0}$, and let $Z_{1}$ the disjoint union of the bibundles $W_{2}^{\times k}$ presenting different compositions of multiplication,

$$
Z_{1}=W_{1} \sqcup W_{2} \sqcup\left(W_{2} \sqcup W_{2}^{\times 2} \sqcup W_{2}^{\times 2} \sqcup W_{2}^{\times 3}\right) \sqcup \ldots
$$

This construction is explained by the following picture:

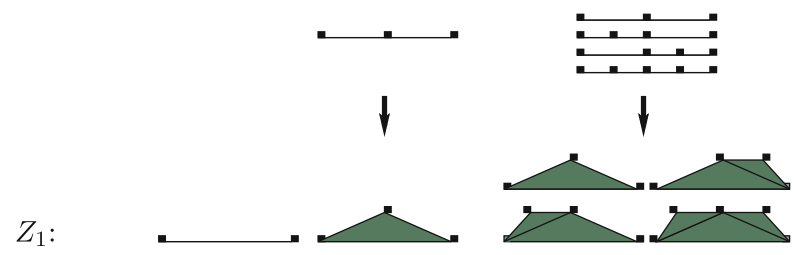

That is, we fill out horns in $\operatorname{Kan}(W)_{1}$ by replacing $W_{1}^{\times n}$ with $W_{2}^{\times(n-1)}$. The projections $Z_{1} \rightarrow K a n(W)_{1}$ and $Z_{1} \rightarrow W_{1}$ are simply the disjoint union of the left and right moment maps, respectively. These projections are both surjective submersions. 
To show that $\tau_{2}(\operatorname{Kan}(W))$ is Morita equivalent to $W$, we only have to show that the pull-back 2-groupoids on $Z$ are the same, that is,

$\operatorname{Kan}(W)_{2} / \sim_{2} \times_{\operatorname{hom}(\partial \Delta[2], K a n(W))} \operatorname{hom}(\partial \Delta[2], Z) \cong W_{2} \times_{\operatorname{hom}(\partial \Delta[2], W)} \operatorname{hom}(\partial \Delta[2], Z)$.

If the map $p: M \rightarrow N$ is surjective and admits local sections at any point in $N$, then the pull-back groupoid $G_{1} \times_{M} N \Rightarrow G_{0} \times_{M} N$ is free and proper if and only if the original groupoid $G_{1} \Rightarrow G_{0}$ is so. Since this is the case, the isomorphism Equation (21) automatically implies that $K a n(W) / \sim_{2}$ is representable. By Proposition 2.4, $\tau_{2}(\operatorname{Kan}(W))$ is a Lie 2-groupoid.

We denote the two pull-backs by the maps $Z_{1} \rightarrow W_{1}$ and $Z_{1} \rightarrow \operatorname{Kan}(W)_{1}$ to $Z_{1}$ by $\left.W\right|_{Z}$ and $\left.\operatorname{Kan}(W)\right|_{Z}$, respectively, we construct morphisms

$$
\pi:\left(\left.K a n(W)\right|_{Z}\right)_{2} \rightarrow\left(\left.W\right|_{Z}\right)_{2}, \quad \iota:\left(\left.W\right|_{Z}\right)_{2} \rightarrow\left(\left.\operatorname{Kan}(W)\right|_{Z}\right)_{2},
$$

and we prove that $\pi \circ \iota=i d$ and $\iota \circ \pi \sim i d$ up to terms in $\left(\left.K a n(W)\right|_{Z}\right)_{3}$. Then, the above isomorphism follows naturally. Observe that usually $\operatorname{Kan}(W)$ is not a Lie 2-groupoid, but the pull-back construction described in Sect. 3.1 is valid also when $X$ is a local Kan manifold. We form $\left(\left.X\right|_{Z}\right)_{n}=\operatorname{hom}\left(s k_{1}(\Delta[n]) \rightarrow \Delta[n], Z \rightarrow\right.$ $X)$, where $s k_{1}$ denotes taking the 1-dimensional skeleton. By [14, Lemma 2.4 ], the $\left(\left.X\right|_{Z}\right)_{n}$ are manifolds. Then it is easy to check that $\tau_{2}\left(\left.\operatorname{Kan}(W)\right|_{Z}\right)=\left.\tau_{2}(\operatorname{Kan}(W))\right|_{Z}$.

We first construct $\iota$. Let $S$ be a simplicial polygon with three marked points, namely a simplicial set constructed inductively

$$
\Delta[2]=S_{0} \hookrightarrow S_{1} \hookrightarrow S_{2} \hookrightarrow \cdots \hookrightarrow S_{i} \cdots,
$$

by push-out, $S_{i+1}=S_{i} \sqcup_{\Delta[1]} \Delta[2]$, and the three marked points are the vertices of $S_{0}$. With these three marked points, the $S_{i}$ 's can be viewed as generalized triangles with their three sides a concatenation of line segments. In this sense, we also have the three face maps $d^{k}$.

There is a natural embedding $W \hookrightarrow K a n(W)$, but this embedding does not yield a map $\left.\left.W\right|_{Z} \rightarrow K \operatorname{an}(W)\right|_{Z}$. In fact, take an element $(w, \partial z) \in\left(\left.W\right|_{Z}\right)_{2}=\sqcup_{i} \operatorname{hom}\left(S_{i}, W\right)$ for a certain set of $S_{i}$ 's, then $(w, \partial z) \notin\left(\left.\operatorname{Kan}(W)\right|_{z}\right)_{2}$ since $\partial w$, the boundary of $w$, is not mapped to $\partial z$ under the map $\partial_{2} Z \rightarrow \partial_{2} \operatorname{Kan}(W)$. Here, $\partial_{k} \square=\operatorname{hom}(\partial \Delta[k], \square)$. To construct $\iota$, we need to inductively construct morphisms $\mu_{i}: \operatorname{hom}\left(S_{i}, W\right) \rightarrow$ $\operatorname{Kan}(W)_{2}$ such that they commute with the face maps $d_{k}$ for $k=0,1,2$,

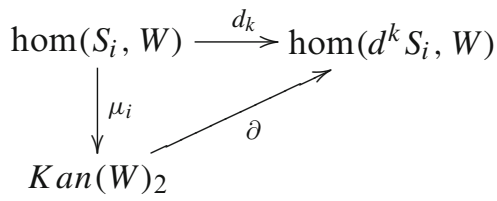

Then, $\iota(w, \partial z):=\left(\mu_{i}(w), \partial z\right)$, where $w \in \operatorname{hom}\left(S_{i}, W\right)$.

Step 1: We first prove the case $i=1$. We simplify the notation by $\operatorname{setting} K:=$ $\operatorname{Kan}(W)$. 


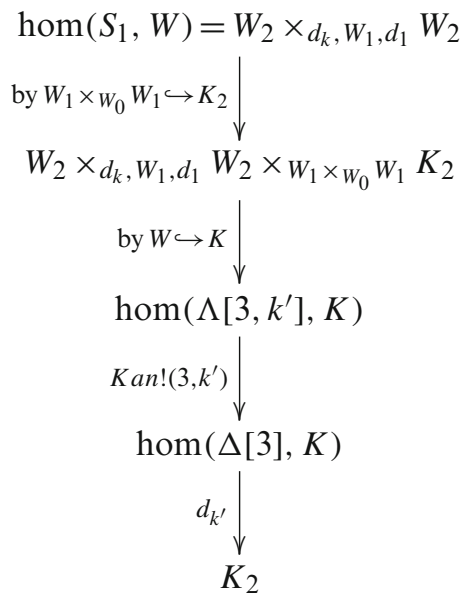

But this map does not commute with the face map (see Equation (22)). To obtain a map that commutes with the face maps, we only need to compose this map with the following one,

$$
\begin{gathered}
K_{2} \rightarrow\left(K_{2} \times W_{1}\left(W_{1} \times W_{0} W_{1}\right)\right) \times{ }_{K_{1} \times W_{0} W_{1}} W_{1} \times W_{0} W_{1} \\
W_{1} \times W_{0} W_{1} \hookrightarrow K_{2} \downarrow \\
\operatorname{hom}\left(\Lambda\left[3, k^{\prime \prime}\right], K\right) \rightarrow K_{2}
\end{gathered}
$$

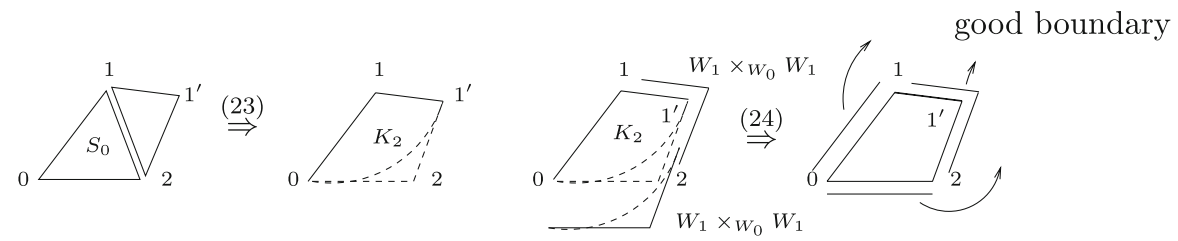

Step 2: Now suppose we have such a map $\operatorname{hom}\left(S_{i}, W\right) \rightarrow K$, then we can construct a map $\operatorname{hom}\left(S_{i+1}, W\right) \rightarrow K$ as follows,

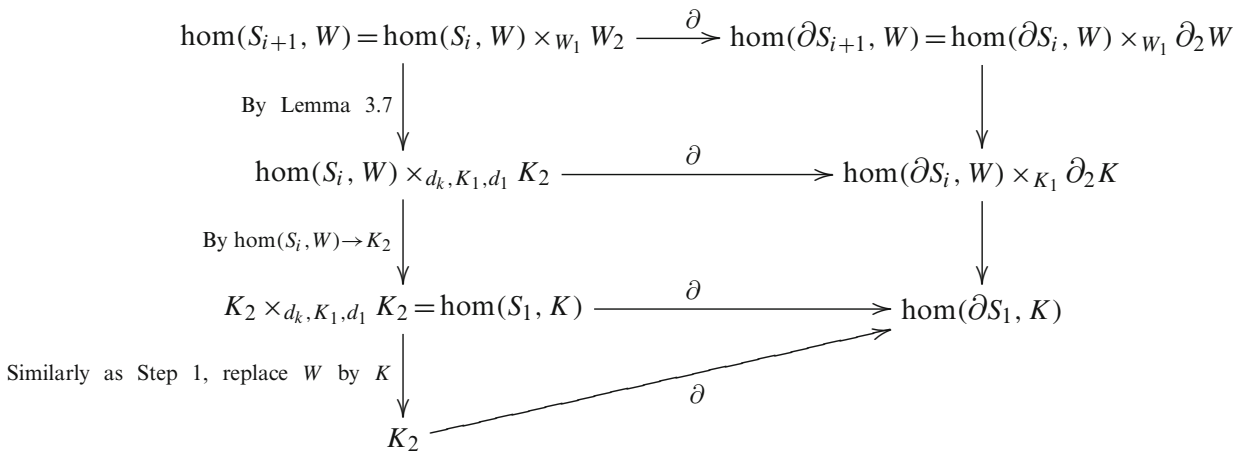




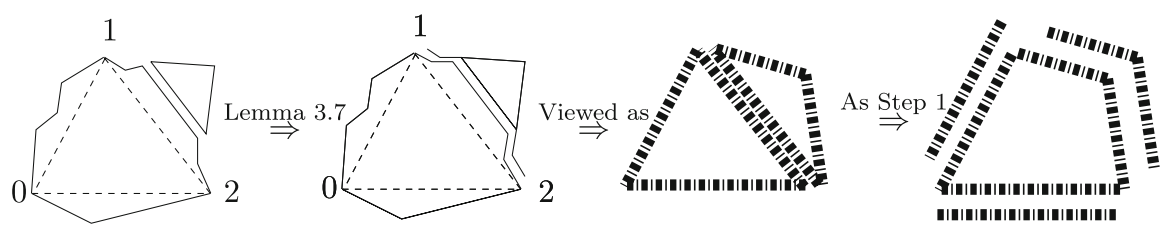

LEMMA 3.7. There is a natural morphism $K_{1} \times{ }_{W_{1}} W_{2} \rightarrow K_{2}$.

We leave the proof of this lemma involving the Kan condition for $K$ to the reader.

To construct $\pi$, we first construct a local morphism $f: \operatorname{Kan}(W) \rightarrow W$ inductively. The first step is to construct $f_{n}^{1}$ by

$$
\begin{aligned}
W_{n}^{1} & =\operatorname{hom}\left(\Delta[n], W \underset{\amalg \Lambda[k, j] \times \operatorname{hom}(\Lambda[k, j], W)}{\left.\coprod_{(k)} \Delta[k] \times \operatorname{hom}(\Lambda[k, j], W)\right) \rightarrow}\right. \\
& \rightarrow W_{n} \coprod_{\cdots} \operatorname{hom}(\Delta[n], \Delta[k]) \times \operatorname{hom}(\Delta[k], W) \rightarrow \\
& \rightarrow W_{n} .
\end{aligned}
$$

In the second step, we use the strict Kan condition $\operatorname{hom}(\Lambda[k, j], W) \cong W_{k}$ when $k \geq 2$, and we choose a local section $\operatorname{hom}(\Lambda[2,1], W) \rightarrow W_{2}$ when $k=2$. The last step follows from the composition $\operatorname{hom}(\Delta[n], \Delta[k]) \times \operatorname{hom}(\Delta[k], W) \rightarrow W_{n}$, and thus both spaces in the push-out have a natural map to $W_{n}$.

Suppose that $f^{\beta}: W^{\beta} \rightarrow W$ is constructed. Then by definition of $W^{\beta+1}$ in Equation (6), $f^{\beta+1}$ is the composition of the following natural morphisms

$$
\begin{aligned}
W_{n}^{\beta+1} & =\operatorname{hom}\left(\Delta[n], W^{\beta} \coprod_{\amalg \Lambda[k, j] \times \operatorname{hom}\left(\Lambda[k, j], W^{\beta}\right)} \Delta[k] \times \operatorname{hom}\left(\Lambda[k, j], W^{\beta}\right)\right) \rightarrow \\
& \rightarrow \operatorname{hom}\left(\Delta[n], W \coprod_{\amalg \Lambda[k, j] \times \operatorname{hom}(\Lambda[k, j], W)} \Delta[k] \times \operatorname{hom}(\Lambda[k, j], W)\right)= \\
& =W_{n}^{1} \stackrel{f_{n}^{1}}{\rightarrow} W_{n} .
\end{aligned}
$$

Then $f$ is the colimit of the $f^{\beta}$.

More geometrically, if we view an element in $\operatorname{Kan}(W)_{2}$ as a set of adjacent small triangles of $W_{2}, f_{2}$ consists in composing these small triangles into a big one in 0 $W_{2}$ with a choice of filling for each $W_{1} \times{ }_{W_{0}} W_{1}$, which is given by $f^{1}$.
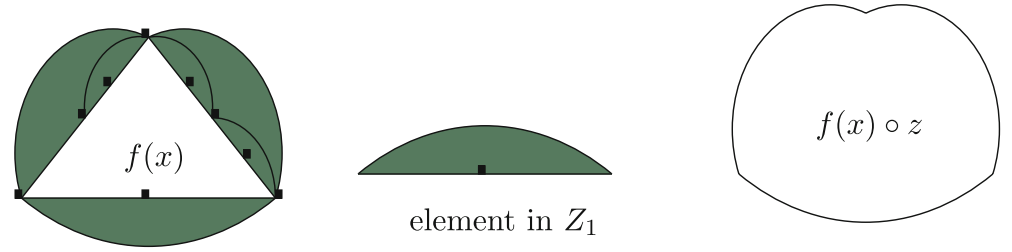
Now, when we make a choice of fillings for a $W_{1} \times W_{0} W_{1}$ on the boundary, instead of choosing some filling given by $f^{1}$, we choose the element $\partial z \in$ $\operatorname{hom}(\partial \Delta[2], Z)$, then this element in $W_{2}$ is denoted by $f_{2}(x) \circ \partial z$.

Thus, $f_{2}$ induces a map

$K a n(W)_{2} \times \operatorname{hom}(\partial \Delta[2], K a n(W)) \operatorname{hom}(\partial \Delta[2], Z) \stackrel{\pi}{\rightarrow} W_{2} \times \operatorname{hom}(\partial \Delta[2], W) \operatorname{hom}(\partial \Delta[2], Z)$.

as $(x, \partial z) \mapsto\left(f_{2}(x) \circ \partial z, \partial z\right)$. In Lemma 3.8, we give a combinatorial proof that this map does not depend on the choice of fillings. Hence, we obtain a well-defined global map $\pi$.

Then it is not hard to see that $\pi \circ \iota=i d$ since $\pi$ is exactly the procedure opposite to $\iota$.

The procedure to form $\pi$ and $\iota$ consists in using $K a n !(3, j)$ to compose [for example Equation (23)], hence $\iota \circ \pi$ and $i d$ differ by terms in $K a n(W)_{3}$.

LEMMA 3.8. The map $\pi$ does not depend on the choice of sections in the construction of $f$.

Proof. We denote an element $\eta \in \operatorname{Kan}(W)_{2}$ by a bicolored tree
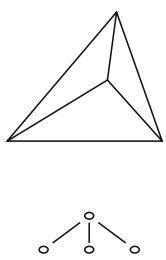
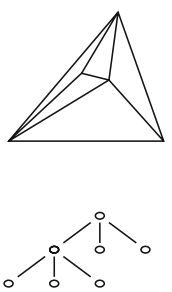
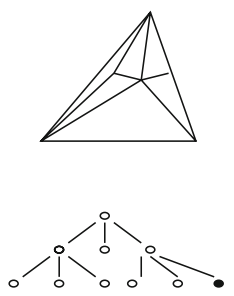
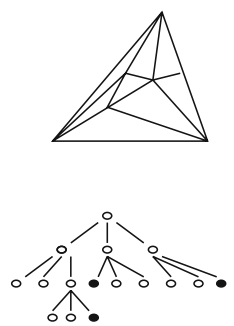

A point is black if it represents a weird triangle, i.e., a triangle that comes from the first copy of $X_{1} \times_{X_{0}} X_{1}$ which serves as an artificial filling; otherwise, it is white. For the other two copies of $X_{1} \times_{X_{0}} X_{1}$, they are degenerated and cannot be glued directly to nondegenerate triangle in $W_{2}$. Since degenerated elements play the role of identities in the composition $\pi$, we shall ignore them here. We now prove the lemma by induction on the number of generations and the number of points in the youngest generation. It is obvious for the initial case.

We take three sibling points in the youngest generation. If all of them are white, then we use $\operatorname{Kan}(3, j)$ without a choice and we obtain an element $\eta^{\prime} \in \operatorname{Kan}(W)_{2}$ which has fewer generations or fewer points in the youngest generation. The result follows in this case.

If one of the three siblings is black, then there is precisely one black one in these three siblings, which we denote by $x$. Since $\operatorname{hom}(\partial \Delta[2], Z)$ will give the fillings for the weird triangles on the border of $\eta$, to show the independence, we only have to 
deal with the inner triangles. Then some ancestor of $x$ must have a black descendant $y$, because a weird triangle must lie on the side of another triangle (which is the parent of $y$ ).

The simplest situation in this case is when the other black descendant is a (true) cousin (namely their direct ancestors are siblings)
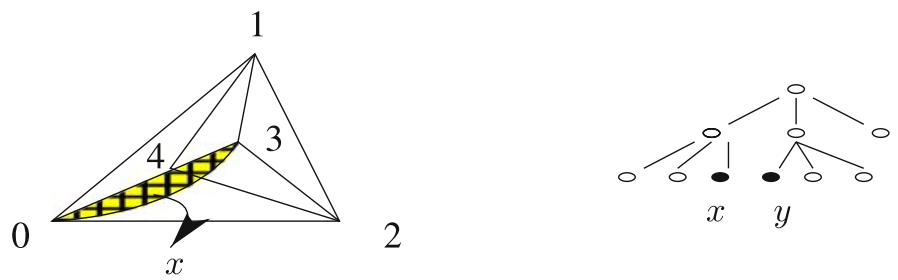

We choose a triangle $\eta_{034}$, and we are given triangles $\eta_{014}, \eta_{134}, \eta_{123}, \eta_{234}, \eta_{024}$. The procedure is to compose $\eta_{034}, \eta_{014}, \eta_{134}$ first to obtain $\eta_{013}$ by $\operatorname{Kan}(3,3)$; then to compose $\eta_{034}, \eta_{024}, \eta_{234}$ to obtain $\eta_{023}$ by $\operatorname{Kan}(3,3)$; finally, to compose $\eta_{013}, \eta_{023}$, and $\eta_{123}$ to obtain $\eta_{012}$ by $\operatorname{Kan}(3,3)$. These can be viewed as multiplications for 2-groupoid [14, Section 2.3]. By the associativity of such multiplications (or equivalently by $\operatorname{Kan}(3, j)$ ! and $\operatorname{Kan}(4, j) !)$, we can obtain the same $\eta_{012}$ by another order of composition, namely we use $\operatorname{Kan}(3,2)$ first to obtain $\eta_{124}$ then $\operatorname{Kan}(3,3)$. Since the second way of composing does not depend on the choice of $\eta_{034}$, our final result $\eta_{012}$ does not depend on the choice either. Hence, by the induction hypothesis, we will obtain an element $f_{2}(x) \circ \partial z$ which is independent of the choices made.

We might meet more complicated situations, namely when the other black descendant $y$ is a more remote cousin, but we can reduce them to the simple situation above:
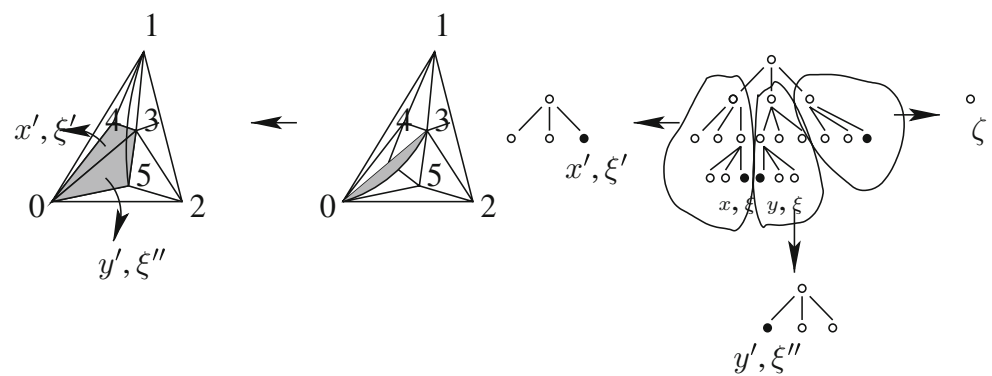

We choose a triangle $\xi \in W_{2}$ corresponding to $x$ in the youngest generation. Then we use $\operatorname{Kan}(3, j)$ to compose to obtain $\xi^{\prime}$ and $\xi^{\prime \prime}$ corresponding to $x^{\prime}$ and $y^{\prime}$ respectively. As shown in the picture, the true cousins $x^{\prime}, y^{\prime}$ are ancestors of $x$ and $y$ respectively. We do the same for the other branch, and obtain $\zeta$. In this procedure, we might have to make other choices of fillings for other black points. But 
it does not matter, since we want to show the independence of the final result on the choice of $\xi$.

We recover the situation of Equation (26). We choose a filling $\eta_{234}$. By $\operatorname{Kan}(4, j)$ ! the final result does not depend on the order of composition. Then by $\operatorname{Kan}(3,2)$, we first obtain $\eta_{124}$; by the induction hypothesis, we obtain $\eta_{024}$ independently of the choice of $\xi$ because $\eta_{024}$ contains fewer descendants than $\eta_{012}$; finally, by $\operatorname{Kan}(3,3)$ we obtain $\eta_{012}$, which is independent of the choice of filling $\xi$.

LEMMA 3.9. If both $X$ and $Y$ are Lie n-groupoids, then a hypercover of local Kan simplicial manifolds $X \stackrel{\sim}{\rightarrow} Y$ is automatically a hypercover of Lie n-groupoids.

Proof. Since $X$ is a Lie $n$-groupoid, there are composed morphisms $\operatorname{hom}(\Lambda[n+$ $1, j], X) \cong X_{n+1} \stackrel{d_{j}}{\rightarrow} X_{n} \rightarrow Y_{n}$ and $\operatorname{hom}(\Lambda[n+1, j], X) \cong X_{n+1} \stackrel{d_{j}}{\rightarrow} X_{n} \stackrel{\partial}{\rightarrow} \operatorname{hom}(\partial \Delta[n]$, $X)$. These maps yield a map, $\operatorname{hom}(\Lambda[n+1, j], X) \stackrel{p}{\rightarrow} \operatorname{hom}(\partial \Delta[n] \rightarrow \Delta[n], X \rightarrow Y)$. With this map, we rewrite

$\operatorname{hom}(\partial \Delta[n+1] \rightarrow \Delta[n+1], X \rightarrow Y) \cong \operatorname{hom}(\Lambda[n+1, j], X) \times_{\operatorname{hom}(\partial \Delta[n] \rightarrow \Delta[n], X \rightarrow Y)} X_{n}$.

Since $X \stackrel{\sim}{\rightarrow} Y$ as local Kan simplicial manifolds, the following map

$$
X_{n+1} \cong \operatorname{hom}(\Lambda[n+1, j], X) \rightarrow \operatorname{hom}(\Lambda[n+1, j], X) \times_{\operatorname{hom}(\partial \Delta[n] \rightarrow \Delta[n], X \rightarrow Y)} X_{n}
$$

is a surjective submersion. This implies that $X_{n} \rightarrow \operatorname{hom}(\partial \Delta[n] \rightarrow \Delta[n], X \rightarrow Y)$ is injective. However, $X_{n} \rightarrow \operatorname{hom}(\partial \Delta[n] \rightarrow \Delta[n], X \rightarrow Y)$ is a surjective submersion by the condition of hypercovers. Hence, $X_{n} \cong \operatorname{hom}(\partial \Delta[n] \rightarrow \Delta[n], X \rightarrow Y)$, which shows that $X \stackrel{\sim}{\rightarrow} Y$ as Lie $n$-groupoids.

This lemma implies

COROLLARY 3.10. Two Lie n-groupoids $X$ and $Y$ are Morita equivalent as local Kan simplicial manifolds if and only if they are Morita equivalent as Lie n-groupoids.

THEOREM 3.11. If $\phi: X \stackrel{\sim}{\rightarrow} Y$ is a hypercover of local Kan simplicial manifolds, and if $\operatorname{Kan}(X)_{2} / \sim_{2}$ is representable, then both $\tau_{2}(\operatorname{Kan}(X))$ and $\tau_{2}(\operatorname{Kan}(Y))$ are Lie 2 -groupoids and the induced map $\tau_{2}(K a n(X)) \rightarrow \tau_{2}(K a n(Y))$ is a hypercover of Lie 2-groupoids.

Proof. We first show that if $\phi: K \rightarrow K^{\prime}$ is a hypercover of Kan simplicial sets (i.e., if the map in Equation (20) is only assumed to be surjective), then the natural map

$$
\tau_{n}(K)_{n} \stackrel{f}{\rightarrow} \operatorname{hom}\left(\partial \Delta[n] \rightarrow \Delta[n], \tau_{n}(K) \rightarrow \tau_{n}\left(K^{\prime}\right)\right),
$$


is an isomorphism. Observe that the right-hand side is simply hom $(\partial \Delta[n], K)$ $\times_{\operatorname{hom}\left(\partial \Delta[n], K^{\prime}\right)} \operatorname{hom}\left(\Delta[n], \tau_{n}\left(K^{\prime}\right)\right)$. Thus, we have a commutative diagram

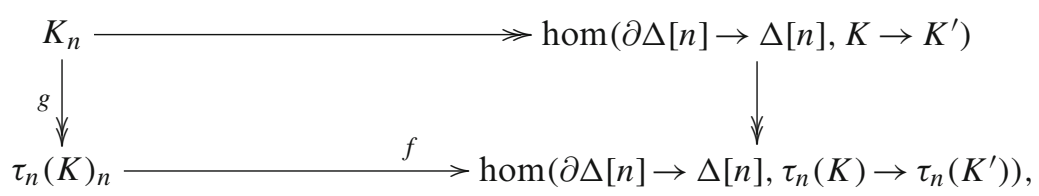

where $\rightarrow$ denotes surjective maps. Then $f$ must be surjective because the fact that $f \circ g$ is surjective implies that $f$ is surjective. Take $\left(\delta x_{n},\left[y_{n}\right]\right) \in \operatorname{hom}(\partial \Delta[n]$, $K) \times_{\operatorname{hom}\left(\partial \Delta[n], K^{\prime}\right)} \operatorname{hom}\left(\Delta[n], \tau_{n}\left(K^{\prime}\right)\right)$. If both $\left[x_{n}\right],\left[x_{n}^{\prime}\right] \in \tau_{n}(K)_{n}$ are mapped to $\left(\delta x_{n},\left[y_{n}\right]\right)$, that is the boundary $\partial x_{n}=\partial x_{n}^{\prime}=\delta x_{n} \in \operatorname{hom}(\partial \Delta[n], K)$ and $\phi_{n}\left(x_{n}^{\prime}\right)=$ $y_{n}^{\prime} \sim y_{n} \sim y_{n}^{\prime \prime}=\phi_{n}\left(x_{n}\right)$, then $y_{n}^{\prime \prime}$ and $y_{n}^{\prime}$ differ by an element $y_{n+1} \in K_{n+1}^{\prime}$. Since

$$
K_{n+1} \rightarrow \operatorname{hom}\left(\partial \Delta[n+1] \rightarrow \Delta[n+1], K \rightarrow K^{\prime}\right),
$$

is surjective, there exists $x_{n+1}$ such that $\phi_{n+1}\left(x_{n+1}\right)=y_{n+1}$ and $x_{n}, x_{n}^{\prime}$ differ by $x_{n+1}$. This proves that $\left[x_{n}\right]=\left[x_{n}^{\prime}\right] \in \tau_{n}(K)_{n}$. Hence, $f$ is also injective.

If the map $p: M \rightarrow N$ is surjective and admits local sections at any point in $N$, then the pull-back groupoid $G_{1} \times{ }_{M} N \Rightarrow G_{0} \times_{M} N$ is free and proper if and only if the original groupoid $G_{1} \Rightarrow G_{0}$ is so. Since this is the case, the isomorphism Equation (27), when applied to $n=2$ and $K=\operatorname{Kan}(X), K^{\prime}=\operatorname{Kan}(Y)$, implies that $\operatorname{Kan}(Y) / \sim_{2}$ is representable. Hence, $\tau_{2}(\operatorname{Kan}(X))$ and $\tau_{2}(\operatorname{Kan}(Y))$ are Lie 2-groupoids by Proposition 2.4.

Now, we only need to verify that the morphism

$$
\tau_{2}(\operatorname{Kan}(X))_{m} \rightarrow \operatorname{hom}\left(\partial \Delta[m] \rightarrow \Delta[m], \tau_{2}(\operatorname{Kan}(X)) \rightarrow \tau_{2}(\operatorname{Kan}(Y))\right)
$$

is a surjective submersion for $m=0,1$. For $m=0$, the result is implied by the fact that $X_{0} \rightarrow Y_{0}$ is a surjective submersion. For $m=1$, by induction, we need to show that the natural map

$$
X_{1}^{\beta+1} \rightarrow \operatorname{hom}\left(\partial \Delta[1] \rightarrow \Delta[1], X^{\beta+1} \rightarrow Y^{\beta+1}\right),
$$

is a surjective submersion supposing that the same is true for $\beta$. We have

$$
X_{1}^{\beta+1}=X_{1}^{\beta} \sqcup \operatorname{hom}\left(\Lambda[2,1], X^{\beta}\right), \quad Y_{1}^{\beta+1}=Y_{1}^{\beta} \sqcup \operatorname{hom}\left(\Lambda[2,1], Y^{\beta}\right) .
$$

The right hand side of Equation (28) decomposes into two terms $I, I I$ according to the decomposition of $Y_{1}^{\beta+1}$,

$$
\begin{aligned}
I & =\operatorname{hom}\left(\partial \Delta[1], X^{\beta}\right) \times_{\operatorname{hom}\left(\partial \Delta[1], Y^{\beta}\right)} \operatorname{hom}\left(\Delta[1], Y^{\beta}\right), \\
I I & =\operatorname{hom}\left(\partial \Delta[1], X^{\beta}\right) \times_{\operatorname{hom}\left(\partial \Delta[1], Y^{\beta}\right)} \operatorname{hom}\left(\Lambda[2,1], Y^{\beta}\right) .
\end{aligned}
$$


By the induction hypothesis, $X_{1}^{\beta} \rightarrow I$ is a surjective submersion. Furthermore, by [14, Lemma 2.5] (in the case $S=T=\Lambda[2,1]$, and $T^{\prime}=\partial \Delta[1]$ ),

$$
\operatorname{hom}\left(\Lambda[2,1], X^{\beta}\right) \rightarrow I I
$$

is a surjective submersion. Thus, Equation (28) is a surjective submersion.

\section{References}

1. Artin, M., Grothendieck, A., Verdier, J.L.: Théorie des topos et cohomologie étale des schémas. Tome 1: Théorie des topos. Lecture Notes in Mathematics, vol. 269, pp. 1963-1964. Springer, Berlin (1972). Séminaire de Géométrie Algébrique du BoisMarie, (SGA 4). Avec la collaboration de N. Bourbaki, P. Deligne et B. Saint-Donat

2. Cattaneo, A.S., Felder, G.: Poisson sigma models and symplectic groupoids. In: Quantization of singular symplectic quotients. Progress in mathematics, vol. 198, pp. 61-93. Birkhäuser, Basel (2001)

3. Crainic, M., Fernandes, R.L.: Integrability of Lie brackets. Ann. Math. (2) 157(2), 575-620 (2003)

4. Friedlander, E.M.: Étale homotopy of simplicial schemes. Annals of Mathematics Studies, vol. 104. Princeton University Press, Princeton (1982)

5. Getzler, E.: Lie theory for nilpotent L-infinity algebras. arxiv:math.AT/0404003

6. Henriques, A.: Integrating $L_{\infty}$-algebras. Compos. Math. 144(4), 1017-1045 (2008)

7. Lurie, J.: Higher topos theory. arXiv:math/0608040v4 [math.CT]

8. May, J.P.: Simplicial objects in algebraic topology. Chicago Lectures in Mathematics. University of Chicago Press, Chicago (1992). Reprint of the 1967 original

9. Mrčun, J.: Stablility and invariants of Hilsum-Skandalis maps. Ph.D. thesis, Utrecht University, Utrecht (1996)

10. Quillen, D.G.: Homotopical algebra. Lecture Notes in Mathematics, vol. 43. Springer, Berlin (1967)

11. Tseng, H.-H., Zhu, C.: Integrating Lie algebroids via stacks. Compos. Math. 142(1), 251-270 (2006)

12. van Est, W.T., van der Lee, M.A.M.: Enlargeability of local groups according to $\mathrm{Mal}^{\prime}$ cev and Cartan-Smith. In: Actions hamiltoniennes de groupes. Troisième théorème de Lie (Lyon, 1986), Travaux en Cours, vol. 27, pp. 97-127. Hermann, Paris (1988)

13. Ševera, P.: L infinity algebras as 1-jets of simplicial manifolds (and a bit beyond). arXiv:math/0612349 [math.DG]

14. Zhu, C.: $n$-Groupoids and stacky groupoids. arxiv:math.DG/0801.2057[math.DG]

15. Zhu, C.: Lie II theorem for Lie algebroids via stacky Lie groupoids. arxiv:math/ 0701024 [math.DG]

16. Zhu, C.: Lie $n$-groupoids and stacky Lie groupoids. arxiv:math.DG/0609420 